Rev Biomed 2004; 15:165-172.

\title{
Efecto de las aguas residuales hospitalarias sobre los patrones de resistencia a antibióticos de Escherichia coli $y$ Aeromonas
}

\section{Artículo Original}

sp.

Edgardo Tzoc ${ }^{1}$, María Laura Arias ${ }^{1 *}$, Carmen Valiente ${ }^{2}$.

${ }^{1}$ Facultad de Microbiología, Universidad de Costa Rica, San José, Costa Rica, ${ }^{2}$ Laboratorio Central, Instituto Costarricense de Acueductos y Alcantarillados, Tres Ríos, Costa Rica.

\section{RESUMEN.}

Introducción. Los centros de atención en salud, especialmente los hospitales, constituyen importantes puntos de origen de descargas de antibióticos hacia el ambiente, produciendo un fuerte impacto en la composición física, química y biológica de los cuerpos receptores.

Material y métodos. Con el fin de evaluar el impacto de un efluente hospitalario sobre los patrones de resistencia a antibióticos de poblaciones bacterianas presentes en agua fresca, se recolectaron muestras de agua a partir de una quebrada aledaña a un hospital clase A en San José, Costa Rica, antes del efluente y después de éste. Se aislaron 120 muestras de Escherichia coli y 75 de Aeromonas sp., a las cuales se les evaluó su patrón de susceptibilidad a antibióticos utilizando la técnica de Kirby Bauer.

Resultados. La más alta prevalencia de resistencia en $E$. coli se obtuvo para dos antibióticos relacionados, ampicilina y amoxicilina, con un porcentaje de 57 y 45 respectivamente. Asimismo, es significativo el nivel de resistencia encontrado para tetraciclina. Con respecto a los aislamientos de
Aeromonas sp. los mayores porcentajes se obtuvieron para esos mismos antibióticos, muy probablemente debido a una resistencia intrínseca de estas bacterias hacia estos b-lactámicos.

Discusión. El efluente hospitalario muestra un importante efecto sobre la presencia de cepas resistentes, aun cuando no es la única fuente de ésta, ya que los patrones de resistencia del cuerpo de agua antes de recibir la descarga hospitalaria muestran ya altos niveles de multirresistencia.

(Rev Biomed 2004; 15:165-172)

Palabras clave: Antibióticos, resistencia antimicrobiana, Escherichia coli, Aeromonas sp., aguas residuales.

\section{SUMMARY.}

Effects of hospitalary waste water residues on the antibiotic resistance patterns of Escherichia coli and Aeromonas sp.

Introduction. Health attention centers, especially hospitals, represent important sources of antibiotic discharges into the environment, producing a big effect

Solicitud de sobretiros: María Laura Arias-Echandi. Facultad de Microbiología, Universidad de Costa Rica, 2060 San José, Costa Rica, C.A. Fax: (506) $2075440 . \quad$ Correo electrónico: mlarias@cariari.ucr.ac.cr

Recibido el 1/Marzo/2004. Aceptado para publicación el 18/Junio/2004.

Este artículo está disponible en http://www.uady.mx/sitios/biomedic/revbiomed/pdf/rb041534.pdf

Vol.15/No.3/Julio-Septiembre, 2004 


\section{E Tzoc, ML Arias, C Valiente.}

on the physical, chemical, and biological composition of receptor bodies.

Material and methods. In order to assess the impact of a hospital effluent on the antibiotic resistance of freshwater bacterial populations, water samples were collected, upstream and downstream, from a creek bordering a Class A hospital, in San José, Costa Rica. 120 samples of Escherichia coli and 75 Aeromonas $\mathrm{sp}$ strains were evaluated and tested for antibiotic susceptibility by the Kirby-Bauer methodology.

Results. Most E. coli strains were resistant to ampicillin and amoxicillin, with a 57 and $45 \%$ resistance, respectively, they also showed significant resistance to tetracycline.

In the Aeromonas sp isolates, the greatest resistance rates were for the same antibiotics as $E$. coli, probably due to the intrinsic resistance of these bacteria to $ß$ lactamics.

Discussion. The hospital effluent shows an important effect on the occurrence of resistant strains, although it is not the only source, since the resistance patterns of the bacteria isolated from the creek before receiving the hospitalary effluent already showed high levels. (Rev Biomed 2004; 15:165-172)

Key words: Antibiotics, antimicrobial resistance, Escherichia coli, Aeromonas sp., waste water.

\section{INTRODUCCIÓN.}

La era de los antibióticos comenzó hace varias décadas y ha sido uno de los más grandes logros en la medicina actual para el control de la mayoría de las enfermedades infecciosas, especialmente las bacterianas. Estos fármacos pueden ser clasificados de varias maneras según su estructura química, su tipo de acción o su espectro de actividad (1).

Actualmente se dispone de una amplia variedad, incluyendo penicilinas, cefalosporinas, tetraciclinas y análogos como las glicilciclinas, aminoglicósidos, monobactam, carbapenem, macrólidos y derivados como los ketolidos, estreptograminas, quinolonas, glicopéptidos, oxazolidinonas, péptidos catiónicos como las bacteriocinas, cecropinas y magaininas (aisladas a partir de bacterias, insectos y piel de ranas), inhibidores de la síntesis del lípido A y los inhibidores de la tRNA sintetasa, como la mupirocina(1).

Las perspectivas para los próximos años en la creación de nuevos antibióticos son amplias debido al estudio extensivo de los genomas bacterianos con el fin de identificar nuevos blancos $(2,3)$ y a diversos factores que tienen influencia en la necesidad de nuevos antibióticos (3).

A pesar de todos estos avances y perspectivas, son muchas las personas que mueren hoy en día debido al problema de la multirresistencia bacteriana a los antibióticos. El uso clínico de los antibióticos comenzó con la aplicación terapéutica de las sulfonamidas en la década de los años 30 (4) y a inicios de los 40 la penicilina se volvió ampliamente disponible en casi todo el mundo (5). Para 1941 casi todas las cepas de Staphylococcus aureus eran susceptibles a la penicilina; no obstante, ya en 1944 se conocen los primeros casos de resistencia por medio de $\beta$ lactamasas (6). Hoy día, prácticamente el $95 \%$ de las cepas son resistentes a penicilina.

Durante los años 40 y principios de los 50 se introdujeron varios tipos de nuevos antibióticos, naturales y semi sintéticos. Sin embargo, esto trajo un problema mayor: el de la multirresistencia. Para 1953, se describió, durante una epidemia en Japón, la primera cepa multirresistente de Shigella (7). Este aislamiento exhibía resistencia a cloranfenicol, tetraciclina, estreptomicina y sulfanilamidas.

Desde entonces esta problemática ha ido en un aumento vertiginoso en todo el mundo, al extremo que para todo tipo de antibiótico ya se han descrito aislamientos de bacterias resistentes, siendo muy probable que pronto se encuentren cepas para las cuales no exista tratamiento alguno, esto debido a la facilidad con que las bacterias adquieren estos factores de resistencia (8).

El uso indiscriminado de antibióticos en las prácticas médicas, veterinarias y agrícolas resulta en la descarga hacia el ambiente de antibióticos y bacterias resistentes a antibióticos (8-11). Los centros de atención en salud, especialmente los hospitales, constituyen importantes puntos de origen de estos desechos y la mayoría de estas descargas drenan al

\section{Revista Biomédica}




\section{Aguas residuales y resistencia a antibióticos.}

alcantarillado público. Sin embargo, algunos de estos drenan directamente a un cuerpo receptor prácticamente sin ningún tratamiento, produciendo un fuerte impacto en la composición física, química y principalmente biológica de estas columnas de agua (12).

Dentro de esta perspectiva, no es extraño pensar que también los hospitales, y más específicamente sus descargas líquidas, ejerzan una presión selectiva sobre la biota presente en los cuerpos receptores que los reciben, contribuyendo a la selección de microorganismos con patrones de resistencia múltiple a antibióticos, ya sea por la presencia de los antibióticos en las descargas (13-14) o por la transmisión de factores de resistencia hacia las bacterias propias de las aguas superficiales (7, 16-18).

Costa Rica no se escapa a esta realidad, razón por la que el objetivo del presente trabajo es analizar el posible impacto de las descargas líquidas de un hospital clase A sobre el patrón de resistencia a antibióticos de cepas de Escherichia coli, y Aeromonas sp. aisladas a partir de una quebrada aledaña. Se utilizaron estos dos géneros de bacterias por ser la primera, representativa de la biota indígena del tracto gastrointestinal de animales y humanos y, por lo tanto, el indicador clásico de contaminación fecal (18). La segunda, por ser una bacteria típica de ambientes acuáticos relacionada con brotes de enfermedades transmitidas por el agua.

\section{MATERIAL Y MÉTODOS.}

Recolección de muestras: durante los meses de febrero, marzo y abril de 2001, se recolectaron 50 muestras de agua de cada uno de tres puntos de muestreo definidos en una quebrada aledaña a un hospital clase A costarricense. Estos puntos incluyen la quebrada $25 \mathrm{~m}$ antes de la confluencia con el efluente, el efluente del hospital y la quebrada $500 \mathrm{~m}$ después de la confluencia con el efluente.

La recolección y transporte de las muestras se realizó de acuerdo a las recomendaciones dadas en Standard Methods for the Examination of Water and Wastewater 2000 (19).
Aislamiento de Escherichia coli y Aeromonas sp: cada muestra recolectada fue inoculada en placas de agar Mc Conkey Sorbitol suplementado con BCIG (ácido 5-bromo, 4-cloro, 3-indolil, b-D glucurónico), e incubado a $37^{\circ} \mathrm{C}$ por 24 horas para el aislamiento de $E$. coli y en placas de agar GSP para el aislamiento de Aeromonas sp En ambos casos se seleccionaron las colonias típicas a las cuales se les realizó prueba de identificación bacteriana utilizando el sistema API 20Eâ y prueba de susceptibilidad a los antibióticos.

Pruebas de susceptibilidad a antibióticos: se seleccionaron los siguientes antibióticos para realizar las pruebas de susceptibilidad, de acuerdo al listado de medicamentos más utilizados en el hospital evaluado: ampicilina $10 \mu \mathrm{g}$, amoxicilina $30 \mu \mathrm{g}$, cefotaxime $30 \mu \mathrm{g}$, gentamicina $10 \mu \mathrm{g}$, amikacina 30 $\mu \mathrm{g}$, tetraciclina $30 \mu \mathrm{g}$, eritromicina $15 \mu \mathrm{g}$, cloranfenicol $30 \mu \mathrm{g}$ y trimetroprim sulfametoxazol $25 \mu \mathrm{g}$.

Las pruebas de susceptibilidad se realizaron de acuerdo con el procedimiento de difusión en placa descrito por Kirby y Bauer y utilizando la cepa Escherichia coli ATCC 2592 como control (20).

Brevemente, a partir de cultivos frescos se preparó una suspensión en solución salina estéril $0,85 \%$, ajustado al 0.5 de la escala de McFarland. En un lapso no mayor de 15 minutos se distribuyó ésta uniformemente en agar Mueller Hinton y se colocaron discos de antibióticos de concentración conocida de las casas DIFCO, Becton Dickinson y Sanofi y se incubaron las placas a $35^{\circ} \mathrm{C}$ por 18 a 20 horas. Los microorganismos fueron clasificados como sensibles o resistentes al antibiótico de acuerdo al diámetro de la zona de inhibición alrededor del disco y los estándares de interpretación suplidos por la casa productora de discos. Organismos considerados como intermedios fueron clasificados como resistentes.

\section{Análisis estadístico.}

Se utilizó el programa SPSS para el análisis estadístico de los datos obtenidos.

\section{RESULTADOS.}

Un total de 120 aislamientos de E. coli y 75 de 
E Tzoc, ML Arias, C Valiente.

\section{Cuadro 1}

Porcentajes totales de resistencia a antibióticos en los aislamientos de Aeromonas sp. y E. coli.

\begin{tabular}{llllllllll}
\hline & E & AK & Amp & Amc & Sxt & Gm & Cl & Te & Ctx \\
\hline Aeromonas sp & 100 & 16 & 91 & 89 & 44 & 11 & 15 & 32 & 31 \\
E.coli & 100 & 5.5 & 57 & 45 & 34 & 14 & 14 & 48 & 12 \\
\hline
\end{tabular}

$\mathrm{E}=$ eritromicina, $\mathrm{AK}=$ amikacina, $\mathrm{Amp}=$ ampicilina, $\mathrm{Amc}=$ amoxicilina, $\mathrm{Sxt}=$ trimetopriím,

$\mathrm{Gm}=$ gentamicina, $\mathrm{Cl}=$ cloranfenicol, $\mathrm{Te}=$ tetraciclina, $\mathrm{Ctx}=$ cefotaxima

Aeromonas sp. fueron evaluados según su patrón de resistencia para cada uno de los antibióticos en estudio.

En el cuadro 1 se muestran los porcentajes totales de resistencia para diferentes clases de antibióticos en los aislamientos de Aeromonas y $E$. coli. En la totalidad de los aislamientos, aparte de la eritromicina (100\%), los b-lactámicos mostraron los mayores niveles de resistencia. La ampicilina resultó ser el antibiótico hacia el cual se detectó un mayor porcentaje de resistencia, tanto en los aislamientos de E. coli como en los de Aeromonas sp con un 91 y un $57 \%$ respectivamente, seguido de la amoxicilina con un porcentaje similar al de la ampicilina en cada bacteria, 89 y $45 \%$. La tetraciclina y el trimetroprim mostraron niveles de resistencia importantes e igualmente significativa también la resistencia detectada para el cefotaxime en Aeromonas sp, no así en E. coli.

Por sitio de muestreo, se observó en ambas bacterias una tendencia al aumento en los niveles de resistencia hacia la mayoría de los antibióticos, especialmente entre los sitios 1 (antes de la descarga) y 2 (descarga del hospital), excepto con respecto a la tetraciclina en los aislamientos de Aeromonas donde se observa una ligera disminución (cuadros 2 y 3 ). En cuanto a los puntos 2 y 3 (después de la descarga) se observa una tendencia al aumento en la resistencia en los aislamientos de Aeromonas sp, excepto para el cloranfenicol donde se obtiene una disminución bastante grande. De hecho, se presenta diferencia estadísticamente significativa $(\mathrm{p}<0.05)$ para amikacina entre los tres sitios de muestreo, para ampicilina entre los sitios 1 y 2 con respecto al 3, para cloranfenicol entre los sitios 2 y 3 , para gentamicina entre los sitios 1 y 2 con respecto al 3 y lo mismo para cefotaxime.

Para E. coli, por el contrario, se observan dos patrones diferentes: aumento o mantenimiento de la resistencia (Ak, Amp, y Te) y disminución de la misma (Amc, Sxt, Gm, Cl y Ctx.). Se obtuvo diferencia estadísticamente significativa para gentamicina en el

Cuadro 2

Porcentajes de resistencia a antibióticos individuales en los aislamientos de Aeromonas sp. en los diferentes puntos de muestreo.

\begin{tabular}{lccccccccc}
\hline & $\mathrm{E}$ & $\mathrm{Ak}^{1}$ & $\mathrm{Amp}^{2}$ & $\mathrm{Amc}$ & $\mathrm{Sxt}$ & $\mathrm{Gm}^{3}$ & $\mathrm{Cl}^{4}$ & $\mathrm{Te}$ & $\mathrm{Ctx}^{5}$ \\
\hline $\begin{array}{l}\text { Sitio } 1 \\
\mathrm{n}=25\end{array}$ & 100 & 0 & 80 & 84 & 32 & 0 & 4 & 32 & 16 \\
Sitio 2 & 100 & 5 & 90 & 90 & 50 & 0 & 35 & 30 & 20 \\
$\mathrm{n}=20$ & 100 & 43 & 100 & 93 & 50 & 27 & 10 & 33 & 50 \\
Sitio 3 & 100 & 16 & 90 & 89 & 44 & 9 & 16 & 32 & 29 \\
$\mathrm{n}=30$ & & & & & & & & & \\
Total & 1075 & & & & &
\end{tabular}

${ }^{1} \mathrm{p}=0.0031$ (entre sitios 2 y 3$), \mathrm{p}=0.0001$ (entre sitios 1 y 3$),{ }^{2} \mathrm{p}=0.0232$ (entre sitios 1,2 y 3$),{ }^{3} \mathrm{p}=0.0002$ (entre sitios $1,2 \mathrm{y}$ $3),{ }^{4} \mathrm{p}=0.0068$ (entre sitios 1 y 2$),{ }^{5} \mathrm{p}=0.0322$ (entre sitios 2 y 3$) \mathrm{p}=0.0030$ (entre sitios 1,2 y 3 )

\section{Revista Biomédica}


Aguas residuales y resistencia a antibióticos.

Cuadro 3

Porcentajes de resistencia a antibióticos individuales en los aislamientos de Escherichia coli en los diferentes puntos de muestreo.

\begin{tabular}{lccccccccc}
\hline & $\mathbf{E}$ & $\mathbf{A k}$ & $\mathbf{A m p}$ & $\mathbf{A m c}$ & $\mathbf{S x t}$ & $\mathbf{G m}^{1}$ & $\mathbf{C l}$ & $\mathbf{T e}^{2}$ & $\mathbf{C t x}$ \\
\hline $\begin{array}{l}\text { Sitio 1 } \\
\mathrm{n}=40\end{array}$ & 100 & 2.5 & 53 & 43 & 33 & 0 & 10 & 35 & 10 \\
$\begin{array}{l}\text { Sitio 2 } \\
\mathrm{n}=40\end{array}$ & 100 & 7.5 & 58 & 48 & 40 & 15 & 23 & 43 & 18 \\
$\begin{array}{l}\text { Sitio 3 } \\
\mathrm{n}=40\end{array}$ & 100 & 7.5 & 60 & 45 & 30 & 10 & 7.5 & 65 & 7.5 \\
$\begin{array}{l}\text { Total n= } \\
120\end{array}$ & 100 & 5.8 & 57 & 45 & 34 & 8 & 14 & 48 & 12 \\
\hline
\end{tabular}

${ }^{1} \mathrm{p}=0.0435$ (entre sitios 2 y 3 ), $\mathrm{p}=0.0060$ (entre sitios 1,2 y 3 ), ${ }^{2} \mathrm{p}=0.0108$ (entre sitios 1 y 2 )

sitio 1 y 2 y para tetraciclina en los sitios 2 y 3 .

En cuanto a los fenotipos de multiresistencia se observa también una tendencia a la acumulación de estos factores entre los puntos 1,2 y 3 de muestreo (cuadro 4).

\section{DISCUSIÓN.}

La presencia y persistencia de bacterias resistentes a antibióticos ha sido descrita en diferentes ambientes incluyendo suelo (21), sedimento (22), agua superficial (23) y agua potable (24), representando un creciente problema de salud pública. Los datos recopilados en el presente trabajo permiten afirmar que en Costa Rica se presenta la misma situación problemática.

De los 75 aislamientos identificados hasta nivel

de género como Aeromonas sp, el 100\% exhiben una resistencia natural hacia la eritromicina, y un porcentaje de resistencia significativo a ampicilina y amoxicilina aun desde el punto 1 de muestreo, anterior al efluente hospitalario. Estos resultados corroboran los obtenidos en aislamientos de Aeromonas de diferente procedencia, como granjas acuícolas (97\%) (25-26) y de procedencia clínica (95\%) (27), al extremo que se piensa en una resistencia natural de las Aeromonas en general hacia estos antibióticos.

Esta bacteria presentó resistencias nulas para los antibióticos amikacina y gentamicina en el punto 1 de muestreo, lo que indica el uso reducido o casi nulo de estos aminoglicósidos por parte de la población general y su uso restringido en el ámbito hospitalario, muy probablemente debido a los efectos tóxicos que

\section{Cuadro 4}

Fenotipos y porcentaje de multiresistencia en los aislamientos de Aeromonas sp. y E. coli, según punto de muestreo.

\begin{tabular}{ccccccc}
\hline $\begin{array}{l}\text { Número de } \\
\text { determinantes } \\
\text { de resistencia }\end{array}$ & $\begin{array}{c}\text { Punto 1 } \\
\text { Aeromonas }\end{array}$ & $\begin{array}{c}E . \\
\text { coli }\end{array}$ & $\begin{array}{c}\text { Punto 2 } \\
\text { Aeromonas } \\
\text { coli }\end{array}$ & $\begin{array}{c}\text { Punto 3 } \\
\text { Aeromonas }\end{array}$ & $\begin{array}{c}E . \\
\text { coli }\end{array}$ \\
\hline $\mathbf{0}$ & 0 & 37.5 & 0 & 27.5 & 0 & 20 \\
$\mathbf{1}$ & 20 & 10 & 10 & 12.5 & 3 & 10 \\
$\mathbf{2}$ & 32 & 17.5 & 30 & 15 & 27 & 27.5 \\
$\mathbf{3}$ ó más & 48 & 45 & 60 & 45 & 70 & 42.5 \\
$\quad$ cepas & 100 & 62.5 & 100 & 72.5 & 100 & 80 \\
resistentes & & & & & & \\
\hline
\end{tabular}




\section{E Tzoc, ML Arias, C Valiente.}

produce su administración, especialmente la gentamicina. Sin embargo, es muy interesante el hecho de que en el punto 3, Aeromonas sp. presenta porcentajes de resistencia significativamente altos para estas sustancias así como para cefotaxime, comprobándose una influencia por parte del efluente hospitalario y la transmisión de factores móviles de resistencia.

Con respecto a de las cepas de E. coli analizadas, se observa también la aparición de porcentajes importantes de resistencia a antibióticos desde el punto 1 . La resistencia más común registrada fue dirigida ampicilina, amoxicilina, trimetroprim sulfa y tetraciclina, presentando en todos los casos porcentajes de resistencia superiores al $30 \%$ en el punto 1 y $40 \%$ en el punto 2 . Los antibióticos beta lactámicos y tetraciclinas son ampliamente utilizados en prácticas humanas y veterinarias, lo cual explica la amplia resistencia observada no sólo en este trabajo, sino en manuscritos similares (9).

Por otro lado, los antibióticos amikacina, gentamicina, cloranfenicol y cefotaxima mostraron porcentajes de resistencia bajos, lo cual es indicativo del poco uso de estos fármacos en el tratamiento indiscriminado de infecciones, tal y como ha sido reportado por diversos autores $(10,28,29)$. No obstante, es importante destacar que la concentración de estos antibióticos aumenta ligeramente en el efluente hospitalario demostrando el impacto de este centro de salud sobre la biota presente en el cuerpo receptor.

Al menos para dos de los antibióticos en estudio, tetraciclina y gentamicina, se pudo comprobar estadísticamente que el vertido del efluente del hospital tiene influencia sobre los patrones de resistencia registrados. La tetraciclina es un antibiótico de uso bastante extendido en medicina y en la industria agropecuaria como promotor del crecimiento y terapia anti-infecciosa, lo cual ha condicionado probablemente la diseminación de cepas resistentes. La resistencia adquirida a este antibiótico es mediada por plásmidos y/o elementos genéticos de transposición, por lo cual la conjugación es uno de los mecanismos involucrados en la transferencia de estos determinantes de resistencia.
Con respecto a la gentamicina, se encontró diferencia estadísticamente significativa entre los puntos 1 y 2, debido al uso restringido de este fármaco al ambiente hospitalario. Sin embargo, el porcentaje de resistencia en el tercer punto de muestreo disminuye probablemente por la pérdida de factores de resistencia o debido a efecto de dilución.

A partir de los aislamientos de ambas bacterias, la investigación de multirresistencia a antibióticos reveló que la resistencia doble y triple es dominante (cuadro 3) y ascendente, similar a resultados obtenidos por Mach y Grimer en 1982 a partir de coliformes aislados de plantas de tratamiento de aguas (17). Es importante destacar que en los puntos 2 y 3 se aislaron cepas con resistencia a cinco o más antibióticos, a diferencia del punto 1 .

Varios autores han establecido que la multirresistencia a antibióticos provee a las cepas ventajas selectivas $(30,31)$ y la evidencia de laboratorio demuestra que bacterias con resistencia a múltiples antibióticos pueden mantener este fenotipo por muchos años en medios de mantenimiento y sin la incorporación de antibióticos (32). En contraste, en un medio donde existe presión hacia la transferencia de genes de resistencia, como es el efluente hospitalario, existe una gran probabilidad de que la eventual reutilización de agua en actividades tan diversas como agricultura, navegación, recreación, pesca, etc., permita la llegada de cepas multirresistentes al ser humano.

La selección y diseminación en la naturaleza de cepas resistentes a los antibióticos es una práctica que se debe evadir, con el fin de mantener un balance ecológico que favorezca el predominio de bacterias susceptibles y asegurar el tratamiento efectivo de las enfermedades infecciosas humanas. De acuerdo a los resultados obtenidos en este estudio, se deduce que el descarte de antibióticos a partir de hospitales directamente a cuerpos receptores favorece la aparición de cepas resistentes, pero hay un efecto importante proveniente del uso indiscriminado de antibióticos en medicina humana, veterinaria y agricultura, entre otros.

\section{Revista Biomédica}




\section{Aguas residuales y resistencia a antibióticos.}

\section{AGRADECIMIIENTO.}

Se agradece al Servicio Alemán de Intercambio Académico por el financiamiento parcial de este proyecto a través de la oficina regional para América Central en San José, Costa Rica. Así mismo se agradece la valiosa colaboración de Laura Villalobos en el desarrollo de este estudio.

\section{REFERENCIAS.}

1.- Bush K. Antimicrobial agents. Curr Op Chem Biol 1997; 1: 169-175.

2.- Allsop A. New antibiotic discovery, novel screens, novel targets, and impact in microbial genomics. Curr Op Microbiol 1998; 1:530-4.

3.- Moir D, Shaw K, Hare R, Vovis G. Genomics and antimicrobial drug discovery. Antimicrob Agents Chemother 1999; 43: 4398-446.

4.- Morris A, Kellner J, Low D. The superbugs: evolution, dissemination and fitness. Curr Op Microbiol 1998; 1: 524-9.

5.- Harrison J, Svec T. The beginning of the end of the antibiotic era? Part 1. The problem: abuse of the "miracle drugs". Quintessence Int 1998; 29: 151-62

6.- Neu H. The crisis of antibiotic resistance. Science 1992; 257: 1064-73.

7.- Roberts M. Tetracycline resistance determinants: mechanism of action, regulation of expression, genetic mobility, and distribution. FEMS Microbial Rev 1996; 19: 124.

8.- Andersson D, Levin B. The biological cost of antibiotic resistance. Curr Op Microbiol 1999; 2: 489-93.

9.- Al-Ghazzi M, Jazrawi S, Al-Doori. Antibiotics resistance among pollution indicator bacteria isolated from Al-Khair river, Baghdad. Wat Res 1988; 2: 641-4.

10.- Al Jebouri M. A note on antibiotic resistance in the bacterial flora of raw sewage and sewage polluted river Tigris in Mosul, Iraq. J Appl Bacteriol 1985; 58: 401-5.

11.- Andersen J, Sandaa R. Distribution of tetracycline resistance determinants among Gram negative bacteria isolated from polluted and unpolluted marine sediments. Appl Environ Microbiol 1994, 60: 908-12.
12.- Hernández A. Caracterización preliminar de la descarga líquida del Hospital México. Tesis. Universidad de Costa Rica. 1999.

13.- Hailling-Sorensen B, Nors S, Lanzky P, Ingerslev F, Holten $\mathrm{H}$, Jorgensen S. Occurrence, fate, and effects of pharmaceutical substances in the environment. A review. Chemosphere 1999; 36: 357-93.

14.- Ternes T. Occurrence of drugs in German sewage treatment plants and rivers. Water Res 1998; 32: 3245-60.

15.- Goñi-Uriza M, Capdepuy M, Arpin C, Raymond N, Caumette P, Quentin C. Impact of an urban effluent on antibiotic resistance of riverine Enterobacteriaceae and Aeromonas spp. Appl Environ Microbiol 2000; 66: 124-32.

16.- Guardabassi L, Petersen H, Olsen J, Dalsguard A. Antibiotic resistance in Acinetobacter spp. isolated from sewers receiving waste effluent from a hospital and a pharmaceutical plant. Appl Environ Microbiol 1998; 64: 3499502 .

17.- Mach P, Grimes J. R-plasmid transfer in a wastewater treatment plant. Appl Environ Microbiol 1982; 44: 1395-403.

18.- Méndez A. Evaluación del patrón de susceptibilidad a antibióticos de bacilos Gram negativos aislados a partir de una quebrada aledaña a un hospital clase A comparada con los aislados a partir de la Quebrada Los Negritos. Tesis. Universidad de Costa Rica, 2000.

19.- Anonymous. Standard Methods for the Microbiological Examination of Water and Wastewater. APHA, Washington D.C., 2000.

20.- Anonymous. Clinical Microbiology Procedures Handbook. ASM, Washington, 1995; p. 460-539.

21.- Trevors J, Berkay T, Bourquin W. Gene transfer among bacteria in soil and aquatic environments. A review. Can J Microbiol 1987; 33: 191-8.

22.- Timoney J, Port J, Giles J, Spanier J. Heavy metal and antibiotic resistance in the bacterial flora of sediments of New York Bight. Appl Environ Microbiol 1978; 36: 409-14.

23.- Winorowski A. Resistance to antibiotics of heavy metal tolerant and heavy metal sensitive bacterial strains. J Envir Sc Hlth 1993; A28: 203-15. 


\section{E Tzoc, ML Arias, C Valiente.}

24.- Moffie B \& Mouton R. Sensitivity and resistance of Legionella pneumophila to some antibiotics and combinations of antibiotics. J Antimicrob Chemother 1988; 22:457-62.

25.- De Paola M, Peeler J, Rodnick G. Effect of oxytetracycline medicated feed on antibiotic resistance of Gram negative bacteria in catfish ponds. Appl Environ Microbiol 1995; 61: 2335-40.

26.- Son R. Antibiotic resistance and plasmid profile of Aeromonas hydrophila isolates from culture fish tilapia (Telapia mossambica). Letters in Applied Microbiol 1997; 24: 479-82.

27.- Chant B, Bolton S. Plasmids and resistance to antimicrobial agents in Aeromonas sobria and Aeromonas hydrophila clinical isolates. Antimicrobic Agents Chemother 1987; 31: 1281-2.

28.- Bhattacherjee J, Spathak J, Gaur A. Antibiotic resistance and metal tolerance of coliform bacteria isolated from Gomati River water at Lucknow city. J Gen Appl Microbiol 1988; 51 : 1285-92.

29.- Pathak S, Bhattachejee J, Ray P. Seasonal variation in survival and antibiotic resistance among various bacterial populations in a tropical river. J Gen Appl Microbiol 1993; 39: 47-56.

30.- Bell J, Elliot G, Smith D. Influence of sewage treatment and urbanization on selection multiple resistance in fecal coliform populations. Appl Environ Microbiol 1983, 46: 22732.

31.- Cohen ML, Tauxe R. Drug resistant Salmonella in the United States: an epidemiological perspective. Science 1986; 234: 964-9.

32.- McKeon D, Calabrese J, Bissonnettes G. Antibiotic resistant Gram negative bacteria in rural ground water supplies. Wat Res 1995; 29:1902-8.

\section{Revista Biomédica}

